\title{
Relationship Between Optimal Gain and Coherence Zone in Flight Simulation
}

\author{
Bruno Jorge Correia Grácio, Ana Rita Valente Paisł M. M. (René) van Paassen \\ and Max Mulder ${ }^{\S}$ \\ Delft University of Technology, Delft, The Netherlands
}

\author{
and \\ Lon C. Kelly, and Jacob A. Houck" \\ NASA Langley Research Center, Hampton, VA
}

\begin{abstract}
In motion simulation the inertial information generated by the motion platform is most of the times different from the visual information in the simulator displays. This occurs due to the physical limits of the motion platform. However, for small motions that are within the physical limits of the motion platform, one-to-one motion, i.e. visual information equal to inertial information, is possible. It has been shown in previous studies that one-to-one motion is often judged as too strong, causing researchers to lower the inertial amplitude. When trying to measure the optimal inertial gain for a visual amplitude, we found a zone of optimal gains instead of a single value. Such result seems related with the coherence zones that have been measured in flight simulation studies. However, the optimal gain results were never directly related with the coherence zones. In this study we investigated whether the optimal gain measurements are the same as the coherence zone measurements. We also try to infer if the results obtained from the two measurements can be used to differentiate between simulators with different configurations. An experiment was conducted at the NASA Langley Research Center which used both the Cockpit Motion Facility and the Visual Motion Simulator. The results show that the inertial gains obtained with the optimal gain are different than the ones obtained with the coherence zone measurements. The optimal gain is within the coherence zone.The point of mean optimal gain was lower and further away from the one-to-one line than the point of mean coherence. The zone width obtained for the coherence zone measurements was dependent on the visual amplitude and frequency. For the optimal gain, the zone width remained constant when the visual amplitude and frequency were varied. We found no effect of the simulator configuration in both the coherence zone and optimal gain measurements.
\end{abstract}

\section{Introduction}

In a driving or flying task in the real world, humans are presented with matching visual and inertial information. However, when researchers try to recreate such conditions in a simulation environment this is normally not true. In flight simulation, pilots are presented with visual information through the simulator displays and inertial information through the simulator motion platform. The visual information shown to

*Ph.D. student, Faculty of Aerospace Engineering, Control and Simulation Division, B.J.CorreiaGracio@tudelft.nl, P.O. Box 5058, 2600 GB Delft, The Netherlands. AIAA Student Member

$\dagger^{\dagger}$ Ph.D. student, Faculty of Aerospace Engineering, Control and Simulation Division, A.R.ValentePais@tudelft.nl, P.O. Box 5058, 2600 GB Delft, The Netherlands. AIAA Student Member.

${ }^{\ddagger}$ Associate Professor, Faculty of Aerospace Engineering, Control and Simulation Division, M.M.vanPaassen@tudelft.nl, P.O. Box 5058, 2600 GB Delft,The Netherlands. AIAA Member.

§Professor, Faculty of Aerospace Engineering, Control and Simulation Division, M.Mulder@tudelft.nl, P.O. Box 5058, 2600 GB Delft, The Netherlands. AIAA Senior Member.

『Software Engineer, Unisys Corporation, Lon.C.Kelly@nasa.gov, NASA Langley Research Center, Hampton, VA 23681-2199.

" Distinguished Research Associate, Simulation Development \& Analysis Branch, Jacob.A.Houck@nasa.gov, NASA Langley Research Center, Hampton, VA 23681-2199. AIAA Associate Fellow. 
the pilot is usually not congruent with the inertial information generated by the motion platform due to its physical limits. Therefore, Motion Cueing Algorithms (MCA) are used to transform aircraft motion into inertial motion that the simulator is able to perform. ${ }^{1}$ The difference between visual information and inertial information should be minimal so that the in-congruency is unnoticed by the pilot in the simulator. ${ }^{2,3}$ If the pilot notices that the inertial information is totally decoupled from what he is seeing on the visual display, immersion in the simulation may be lost $^{3}$ and side effects like motion sickness can occur. ${ }^{4,5}$ However, a situation where the inertial information is equal to the visual information seems also not optimal. Researchers found $^{6-10}$ that subjects judged one-to-one motion, i.e. inertial motion equal to visual motion, as too strong in a simulation environment. A previous study, ${ }^{10}$ where subjects could select the inertial motion amplitude that best matched their visual stimulus, has shown that in a simulator environment, the inertial amplitude selected by subjects as optimal was not equal to the visual amplitude. It was found that the optimal inertial amplitude, hereby defined as optimal gain, depended on the amplitude and frequency of the visual stimulus presented to the participants. Furthermore, the optimal gain selected by subjects depended on the initial amplitude of the inertial motion that was provided. For the same visual condition, initial inertial amplitude higher than the visual amplitude yielded an inertial gain higher than when the initial inertial amplitude was smaller than the visual amplitude. Such results may indicate that there is not just one optimal inertial gain for a certain visual amplitude but a range, or zone, of acceptable inertial motion amplitudes. The range of visual-inertial amplitudes that are considered to be coherent, the so called coherence zone, were measured before $^{3,11,12}$ but no relation has been established between these and an optimal gain zone.

The goal of the present study is two-fold. The first objective is to determine the relationship between the perception coherence zone and the optimal gain. The existence of a zone within which inertial and visual motion are perceived as matching has a direct explanation in human perception mechanism and sensory limitations. The optimal gain, on the other hand, has a much more direct application in flight simulation motion cueing algorithms. The second objective is to make a first step into using the optimal gain and coherence zones measurements as a means to compare different simulators' configurations.

To conduct the study we used the Cockpit Motion Facility (CMF), and the Visual Motion Simulator (VMS) located at NASA Langley Research Center in Hampton VA. An experiment was designed where the optimal gain and the coherence zone measurements were compared within and between the two simulators used in this study.

\section{Method}

\section{II.A. Subjects}

Eight subjects were selected from the employees of the LaRC Flight Simulation Facility. There were seven male and one female participants. The subjects' average age was 49, ranging between 31 and 64 years old. These subjects had varying experience in motion simulators, with one subject having over 40 years and three subjects with over 30 years of experience in cockpit motion systems and three subjects who had never been in a motion simulator before. All subjects were able to complete the required tasks with no discomfort.

\section{II.B. Apparatus}

The Cockpit Motion Facility (CMF), and the Visual Motion Simulator (VMS) located at NASA Langley Research Center in Hampton VA were used to conduct this study.

\section{II.B.1. VMS}

The LaRC VMS, shown in Figure 1, is a generic two person, transport type cockpit permanently mounted on a 60 inch stroke, six degree-of-freedom, synergistic motion platform. For this study, the motion bases were driven in the sway axis. The performance limits of the VMS in the sway axis are: $+/-48 \mathrm{in},+/-24$ $\mathrm{in} / \mathrm{s}$ and $+/-0.6 \mathrm{gs}$. The VMS is equipped with WAC windows, with a horizontal field of view of 65.93 degrees, a vertical field of view of 45.23 degrees and a resolution of 1024 x 944 lines.

\section{II.B.2. $C M F$}

The LaRC CMF (Figure 2) is a novel facility with one motion base and 3 interchangeable cockpits. The motion base is a state-of-the-art, high performance, 76 inch stroke, six degree-of-freedom, synergistic motion 


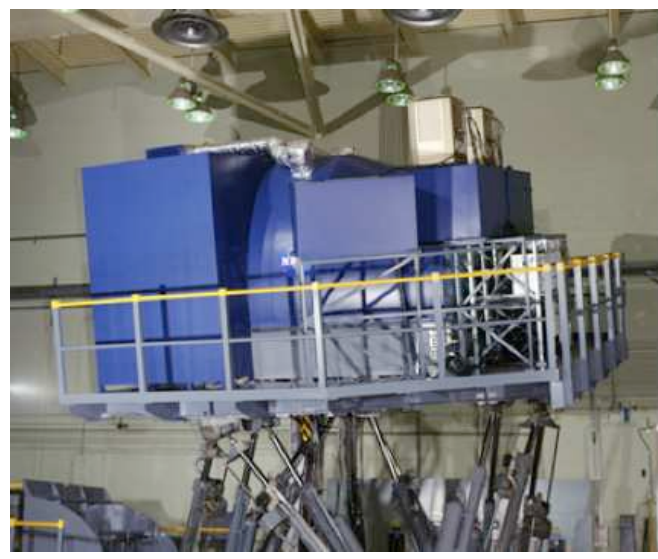

(a) VMS

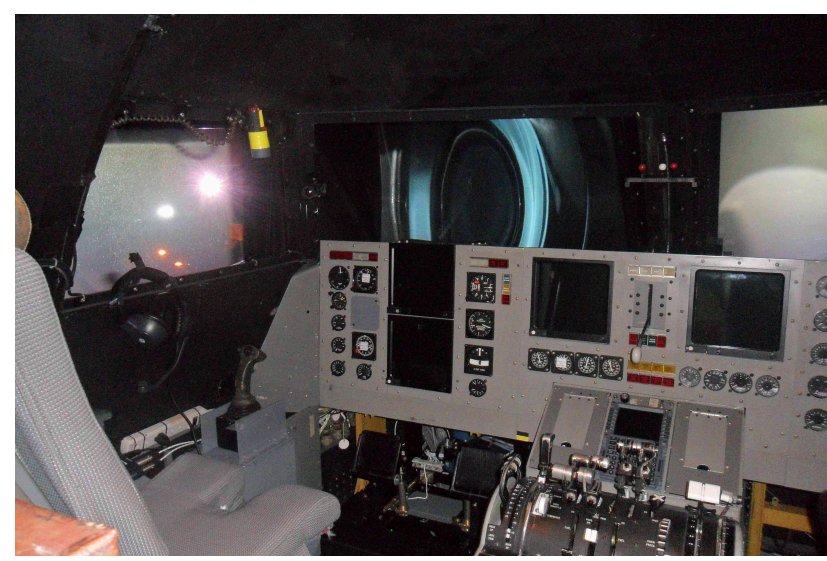

(b) VMS cockpit

Figure 1. The LaRC Visual Motion Simulator.

system. For this study, the Generic Flight Deck (GFD) Cockpit was mounted on the motion base. The GFD cockpit is a futuristic, all glass instrument, easily reconfigurable transport type cockpit with interchangeable and programmable control inceptors. The performance limits of the CMF in the sway axis are $+/-55$ in, $+/-38 \mathrm{in} / \mathrm{sec}$, and +/- $0.7 \mathrm{gs}$. The GFD cockpit also is equipped with WAC windows with a horizontal field of view of 46 degrees and a vertical field of view of 34 degrees and a resolution of $1280 \times 1024$ lines.

In each cockpit (GFD and VMS), the cockpit sound system was used to produce background aero noise to mask any audio cues that might come from the motion base. In both cockpits the trim buttons on top of the side stick were used to increase/decrease self motion, and to indicate when the subject was finished with his/her adjustments.

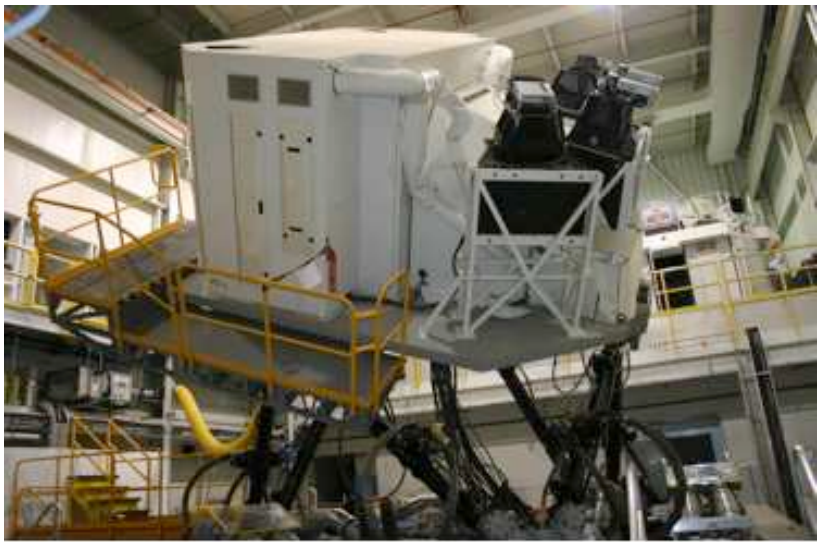

(a) CMF with GFD cabin

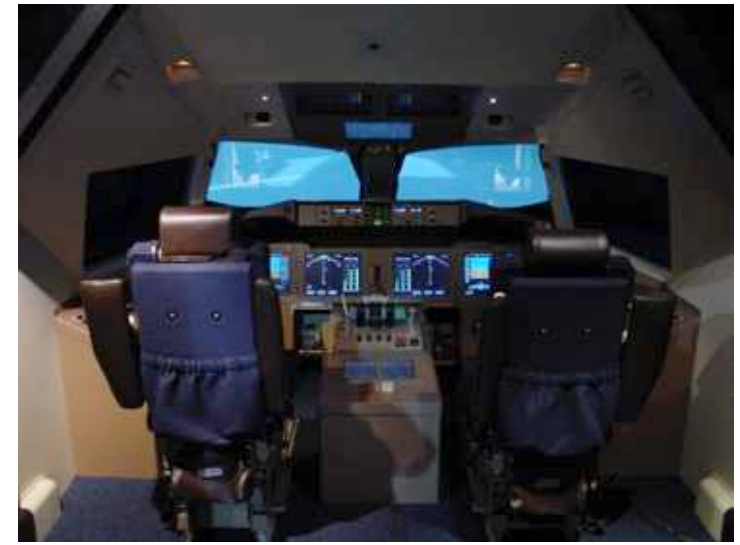

(b) GFD cockpit

Figure 2. The LaRC Cockpit Motion Facility with the Generic Flight Deck Cockpit.

\section{II.C. Experimental Design}

The experiment was divided in two parts. The first part was conducted in the CMF while the second part was conducted in the VMS. Due to simulator scheduling it was not possible to have both simulators available at the same time so randomization between simulators was not conducted. The VMS experimental conditions were performed two months after the CMF experimental conditions. The same subjects were used in the VMS experiment and the experimental order they followed was the same they had in the CMF. Each experiment part was further divided in two blocks.

In the first block all the optimal gain measurements were conducted. Here subjects were asked to adjust the inertial acceleration amplitude of the simulator until the best match between visual and inertial information is obtained. This was done for four different visual signals. The visuals were combinations of 
sinusoids in the lateral direction with amplitudes of 0.5 and $1 \mathrm{~m} / \mathrm{s}^{2}$ and frequencies of 2 and $5 \mathrm{rad} / \mathrm{s}$ for the CMF simulator and 3 and $5 \mathrm{rad} / \mathrm{s}$ for the VMS simulator. Each visual signal was measured for two different initial inertial conditions, the upper optimal gain measurement where the initial inertial amplitude was higher than the visual amplitude and the lower optimal gain measurement where the initial inertial amplitude was lower than the visual amplitude. This creates a total of eight different experimental conditions with three repetitions for each condition. The eight experimental conditions were randomized for each repetition using a latin square design.

In the second experimental block, subjects were instructed to measure the coherence zones. Here subjects have to adjust the inertial amplitude until they find the highest or lowest amplitude that is still coherent with the visual amplitude. The highest inertial amplitude still perceived as coherent is defined as the upper threshold while the lower coherent amplitude is defined as the lower threshold. Subjects conducted upper and lower thresholds measurements using the same four visual profiles of the first block. This led to eight different experimental conditions with three repetitions for each condition. The eight experimental conditions were randomized for each repetition using a latin square design.

The optimal gain experimental block was performed before the coherence zone experimental block for all subjects. This was done to ensure that knowledge regarding what was a coherence zone, which is necessary for the coherence zone instructions, did not influence the optimal gain instructions.

\section{II.D. Procedure}

All subjects started the experiment with the measurement of the optimal gain zone. Each subject was briefed using the standard briefing form. The subject was placed in the pilot side seat, strapped in securely using a standard 5 point harness and instructed to hold his/her head as still as possible against the head rest. Each subject was given several practice runs until he/she felt comfortable with the task. Once the subject was "trained", the experiment started. The experimental run started with a 13 seconds visual and inertial motion with the same frequency and phase but different amplitude. The initial inertial amplitude could be higher or lower than the visual amplitude, depending on the experimental condition that was being measured. This initial inertial amplitude was a random value between 1.4 and 1.6 times the visual amplitude for the upper optimal gain measurement and a random value between 0.4 and 0.6 times the visual amplitude for the lower optimal gain measurement. Subjects where not told if the experimental condition was an upper or lower optimal gain measurement. After the 13 seconds motion, subjects could chose if they wanted to increase/decrease the inertial amplitude. Their task was to find the best inertial amplitude that matched the visual amplitude. The cabin side stick buttons were used to increment/decrement the inertial amplitude. Subjects had visual feedback of the chosen increment/decrement and would press a button in the side stick to send that value to the algorithm. The inertial amplitude of the next run was equal to the inertial amplitude of the previous run plus the number of increments/decrements chosen by the subject times one forty of the visual amplitude. The experimental condition ended when the last three runs had increments/decrements of $1,-1,1$ or $-1,1,-1$. The experimental condition would also stop if subjects failed to converge to a certain value after 30 runs. This procedure was repeated for all the eight different experimental conditions and their repetitions.

Before continuing to the second block, subjects had a small break. Subjects were briefed on how to measure the coherence zone before being placed inside the simulator. After being strapped in the pilot seat, practice runs were performed until subjects felt confident with the coherence zone task. The experiment started by informing subjects if they were measuring an upper or lower threshold. The initial inertial amplitude value was chosen randomly between 0.9 and 1.1 times the visual amplitude. For an upper threshold subjects had to find the highest inertial amplitude that was still coherent with the visual amplitude. For a lower threshold subjects had to find the lowest inertial amplitude that was still coherent with the visual amplitude. Like in the optimal gain measurements, subjects could vary the inertial amplitude at the end of each run by using the buttons in the side stick. The experimental condition would again finish if the subjective input for the last three runs had been $1,-1,1$ or $-1,1,-1$ or if the subject was not able to converge to a value after 30 runs. The measurements would end when all the eight experimental conditions and their repetitions were complete. Each experimental block lasted approximately two hours. 


\section{II.E. Data analysis}

Before analyzing the results, the inertial amplitudes obtained from both experimental blocks were pooled between the three repetitions for each subject. These values were then used for the analysis described below.

As stated before, a previous study ${ }^{10}$ showed that the inertial amplitudes measured when subjects are given the optimal gain instructions led to an optimal gain zone. This zone is defined by the upper and lower optimal gain measurements. In order to compare the optimal gain with the coherence zone we defined for the optimal gain zone a point of mean optimal gain (PMO), which is the middle of the optimal gain zone, and the optimal gain width $(\mathrm{OGW})$. Both the PMO and the OGW are defined by Equations 1 and 2, where $u p_{O G}$ is the upper optimal gain and $l_{O G}$ is the lower optimal gain. For the data analysis of the optimal gain, the dependent variables were the PMO and the OGW while the independent variables where the visual amplitude and frequency.

$$
\begin{gathered}
O G W=u p_{O G}-l_{O G} \\
P M O=l_{O G}+\frac{O G W}{2}
\end{gathered}
$$

From the coherence zone measurements we obtained inertial amplitudes for the upper $\left(t h_{u p}\right)$ and lower $\left(t h_{l o}\right)$ thresholds. From these thresholds we defined the coherence zone width (CZW) and the point of mean coherence (PMC). These were obtained using Equations 3 and 4. For the data analysis of the coherence zone measurements, the dependent variables were the PMC and the CZW while the independent variables where the visual amplitude and frequency.

$$
\begin{gathered}
C Z W=t h_{u p}-t h_{l o} \\
P M C=t h_{l o}+\frac{C Z W}{2}
\end{gathered}
$$

For comparing the coherence zone with the optimal gain we considered that the PMO is equivalent to the PMC and that the OGW is equivalent to the CZW. This can be done since both instructions (coherence zone and optimal gain) measure a zone where there is a relation between visual and inertial information. Therefore we will define the PMO and PMC as point of mean zone (PMZ) measures and the OGW and CZW as zone width (ZW) measures. The PMZs and the ZWs were the dependent variables when comparing the coherence zone with the optimal gain. The independent variables were the instructions, the visual amplitude and the frequency.

When comparing both simulators we again used the PMZs and ZWs as dependent variables and the simulator, instructions and visual amplitude as independent variables.

For the statistical analysis we conducted repeated measures ANOVAs to look for effects of the independent variables in the dependent variables. The statistical analysis were performed with SPSS PASWS 18.0.

\section{Results}

\section{III.A. The CMF simulator}

\section{III.A.1. Optimal Gain}

Figure 3 shows the inertial amplitude values averaged between subjects obtained during the optimal gain measurements. As explained before, the first inertial amplitude provided to the subjects was either much higher (upper optimal gain measurement) or much lower (lower optimal gain measurement) than the visual amplitude. In Figure 3 the upper and lower lines in each plot represent the upper and lower measurements, respectively.

To analyze the optimal gain zone we calculated the point of mean optimal gain (PMO) and the optimal gain width (OGW) given respectively by Equations 2 and 1 . Figure 4 shows the mean values of the obtained $\mathrm{PMO}$ and OGW. A repeated measures ANOVA was conducted to observe the effect of the visual amplitude and frequency on the PMO and OGW. The repeated measures ANOVA results are shown in Table 1.

Figure 4(a) shows that the PMO was significantly higher for the conditions with a visual amplitude of 1 $\mathrm{m} / \mathrm{s}^{2}$. However, the $1 \mathrm{~m} / \mathrm{s}^{2}$ PMOs where further away from the one-to-one line than the $0.5 \mathrm{~m} / \mathrm{s}^{2}$ PMOs. 


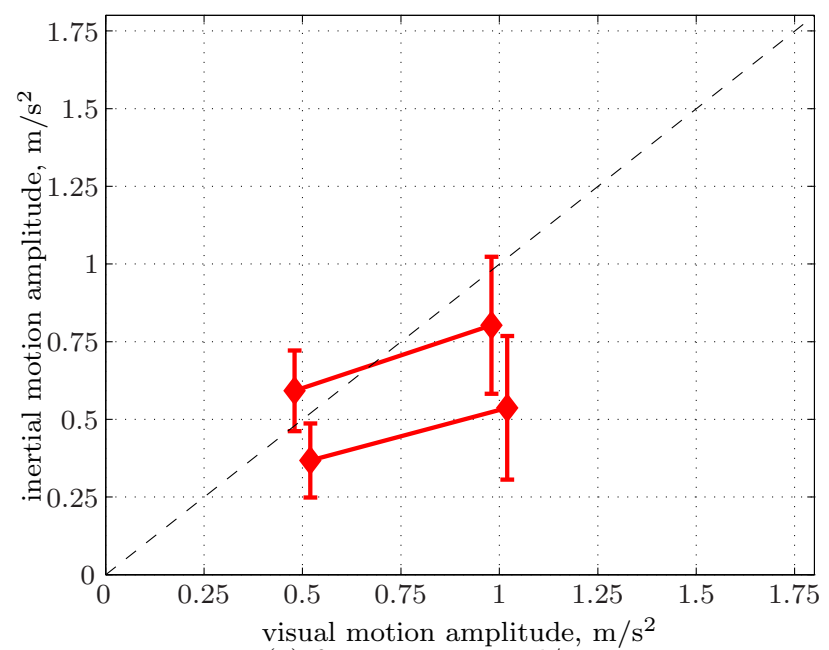

(a) frequency $=2 \mathrm{rad} / \mathrm{s}$

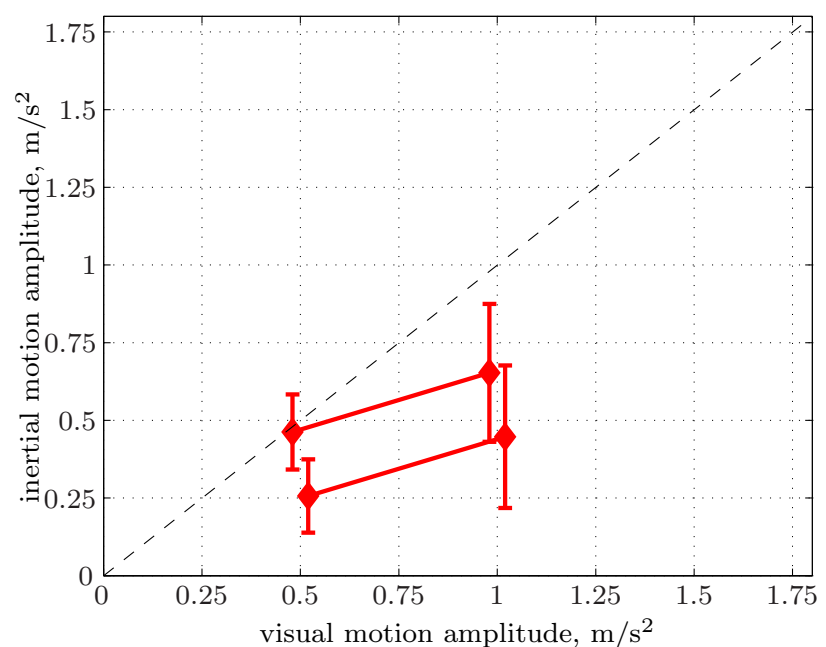

(b) frequency $=5 \mathrm{rad} / \mathrm{s}$

Figure 3. Mean inertial amplitude values for two stimuli frequencies, two visual amplitudes and two different initial inertial conditions. The upper and lower inertial amplitude measurements are represented by the upper and lower lines, respectively. The dashed line shows where the visual motion is equal to the inertial motion. The error bars indicate the $95 \%$ confidence intervals.

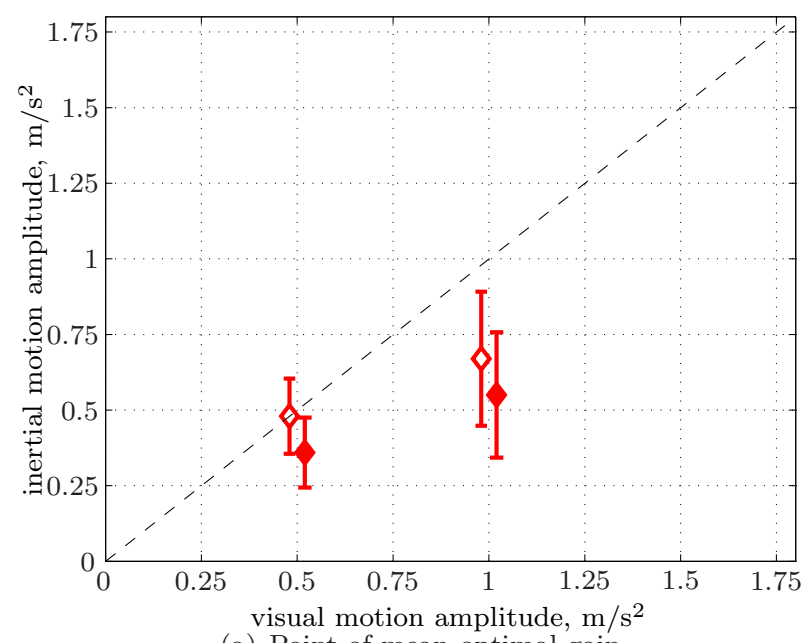

(a) Point of mean optimal gain

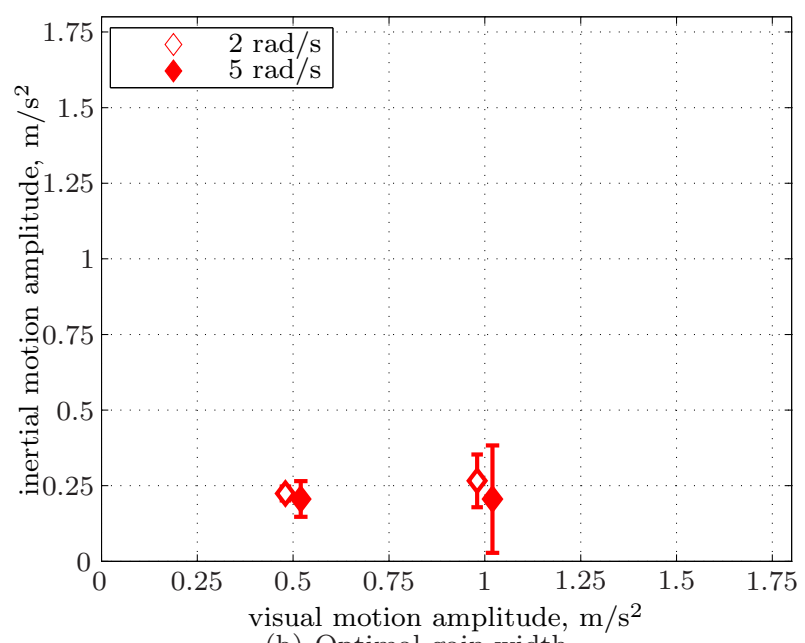

(b) Optimal gain width

Figure 4. Mean PMO and OGW for the optimal gain. The dashed line in Figure 4(a) shows where the visual motion is equal to the inertial motion. The error bars indicate the $95 \%$ confidence intervals.

Table 1. Repeated measures ANOVA results for the point of mean coherence and the coherence zone width.

\begin{tabular}{cccc}
\hline \hline Dependent Variables & Independent Variables & F-ratio & $p$ \\
\hline \multirow{2}{*}{ PMO } & Amplitude & $\mathrm{F}(1,7)=14.87$ & 0.006 \\
& Frequency & $\mathrm{F}(1,7)=13.07$ & 0.009 \\
\hline \multirow{2}{*}{ OGW } & Amplitude & $\mathrm{F}(1,7)=0.19$ & 0.676 \\
& Frequency & $\mathrm{F}(1,7)=1.27$ & 0.298 \\
\hline \hline
\end{tabular}

The PMO was also influenced by the frequency of the visual stimuli. The PMO of the conditions with frequency of $2 \mathrm{rad} / \mathrm{s}$ where higher than the $\mathrm{PMO}$ of the $5 \mathrm{rad} / \mathrm{s}$ condition.

The OGW was not affected by the different visual amplitudes and frequencies (see Table 1). Figure 4(b) shows that the mean OGW remains at the same level, around $0.23 \mathrm{~m} / \mathrm{s}^{2}$, for the tested inertial amplitudes and frequencies. 


\section{III.A.2. Coherence Zone}

Figure 5 shows the mean inertial acceleration values obtained during the coherence zone measurements. The upper threshold is represented by the inertial accelerations above the one-to-one line while the lower threshold is represented by the acceleration values below the one-to-one line.

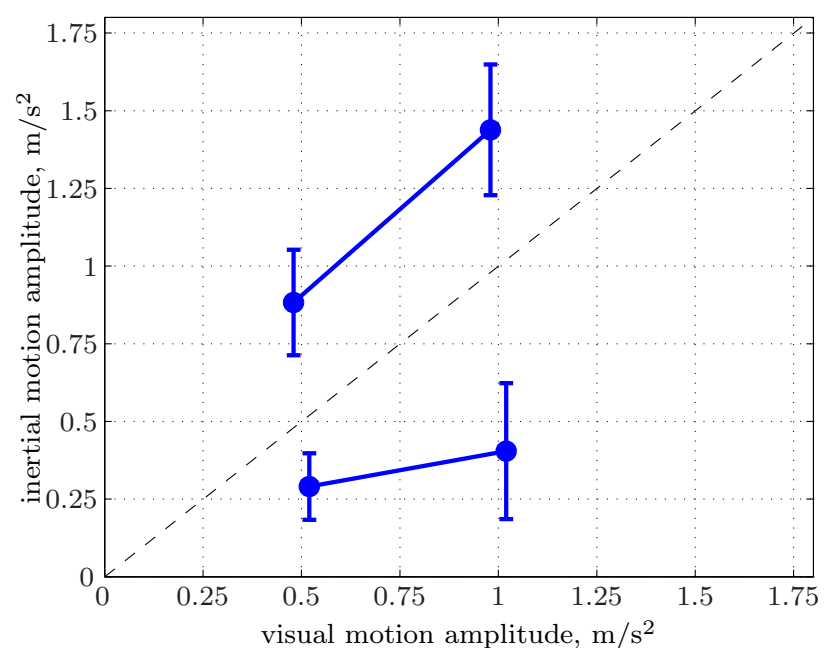

(a) frequency $=2 \mathrm{rad} / \mathrm{s}$

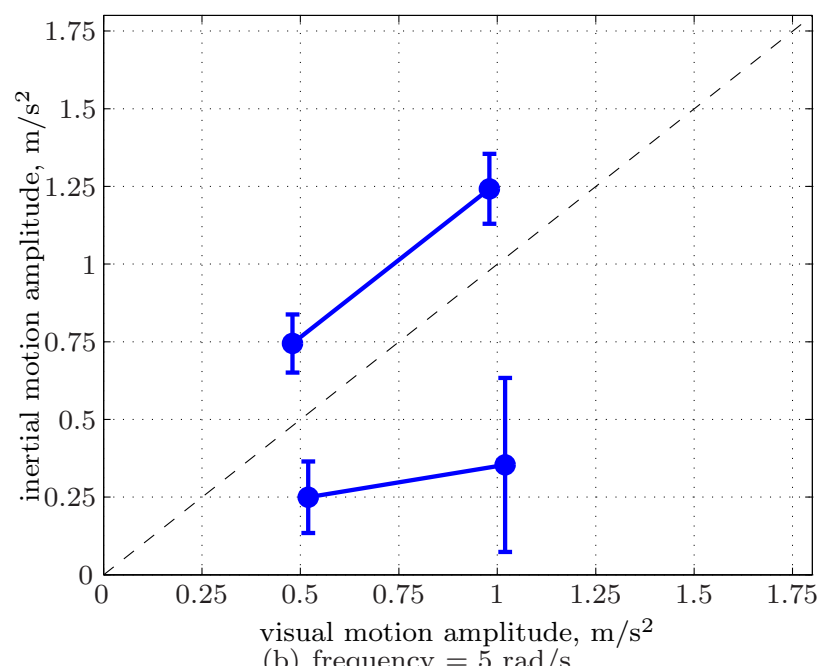

(b) frequency $=5 \mathrm{rad} / \mathrm{s}$

Figure 5. Mean coherence zones for two stimuli frequencies. The dashed line shows where the visual motion is equal to the inertial motion. The error bars indicate the $95 \%$ confidence intervals.

From the upper and lower thresholds we obtained the point of mean coherence (PMC) and the coherence zone width $(\mathrm{CZW})$, see Equations 4 and 3. These measurements were used to analyze the characteristics of the coherence zone, similar to what was done in previous studies. ${ }^{11,12}$

Figure 6 shows the obtained PMC and CZW. A statistical analysis was performed to observe the effect of the visual amplitude and the frequency of the stimuli in both dependent variables. The repeated measures ANOVA results are shown in Table 2 for all the independent variables used in the analysis.

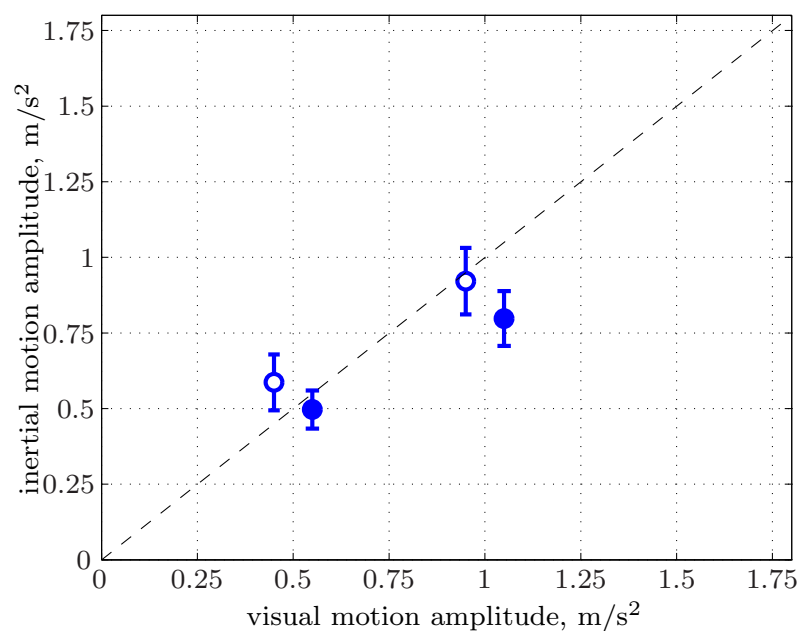

(a) Point of mean coherence

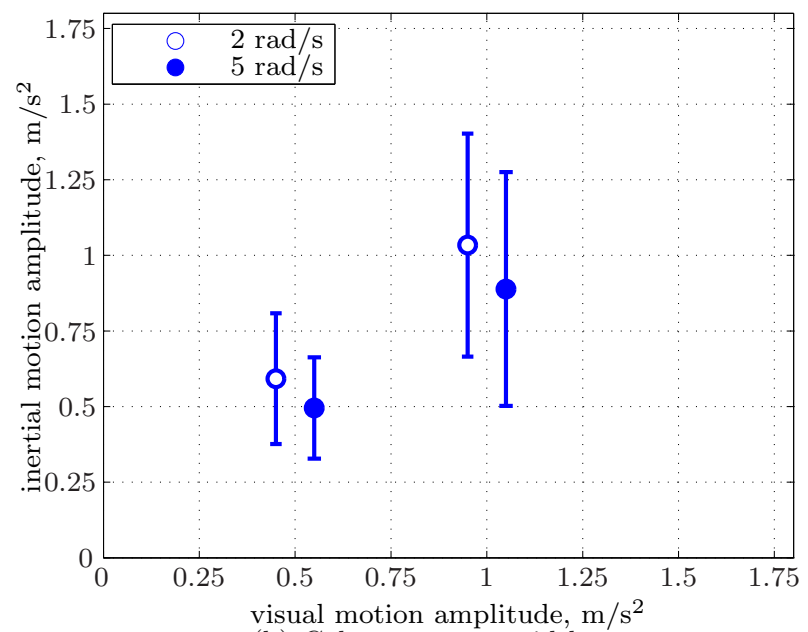

(b) Coherence zone width

Figure 6. Mean PMC and CZW for the coherence zone measurements. The dashed line in Figure 6(a) shows where the visual motion is equal to the inertial motion. The error bars indicate the $95 \%$ confidence intervals.

The PMC was significantly influenced by the visual amplitude and the stimulus frequency. The PMCs of the conditions with higher visual amplitude were significantly higher than the PMCs of the experimental conditions with lower visual amplitude. Such a trend is observed in Figure 6(a). Figure 6(a) also shows that the PMC significantly decreases with an increase in frequency. 
The CZW was significantly higher for the experimental conditions with an higher visual amplitude (Figure 6(b)). The CZW was significantly lower for stimulus with higher frequency content as shown in Figure $6(\mathrm{~b})$.

Table 2. Repeated measures ANOVA results for the point of mean coherence and the coherence zone width.

\begin{tabular}{cccc}
\hline \hline Dependent Variables & Independent Variables & F-ratio & $p$ \\
\hline \multirow{2}{*}{ PMC } & Amplitude & $\mathrm{F}(1,7)=101.20$ & 0.000 \\
& Frequency & $\mathrm{F}(1,7)=7.08$ & 0.032 \\
\hline \multirow{2}{*}{ CZW } & Amplitude & $\mathrm{F}(1,7)=17.66$ & 0.004 \\
& Frequency & $\mathrm{F}(1,7)=9.60$ & 0.017 \\
\hline \hline
\end{tabular}

\section{III.A.3. Optimal Gain versus Coherence Zone}

Figure 7 shows the mean upper and lower thresholds combined with the mean upper and lower optimal gains. Here, we observe that the lower threshold seems similar to the lower optimal gain measurement. However, the upper threshold is higher than the upper optimal gain measurement. As explained before, we used the point of mean zone (PMZ) measures and the zone width (ZW) measures to statistically compare the coherence zone with the optimal gain. The optimal gain PMZ is the PMO whereas for the coherence zone is the PMC. Similarly, the ZW for the optimal gain is the OGW whereas for the coherence zone is the CZW.
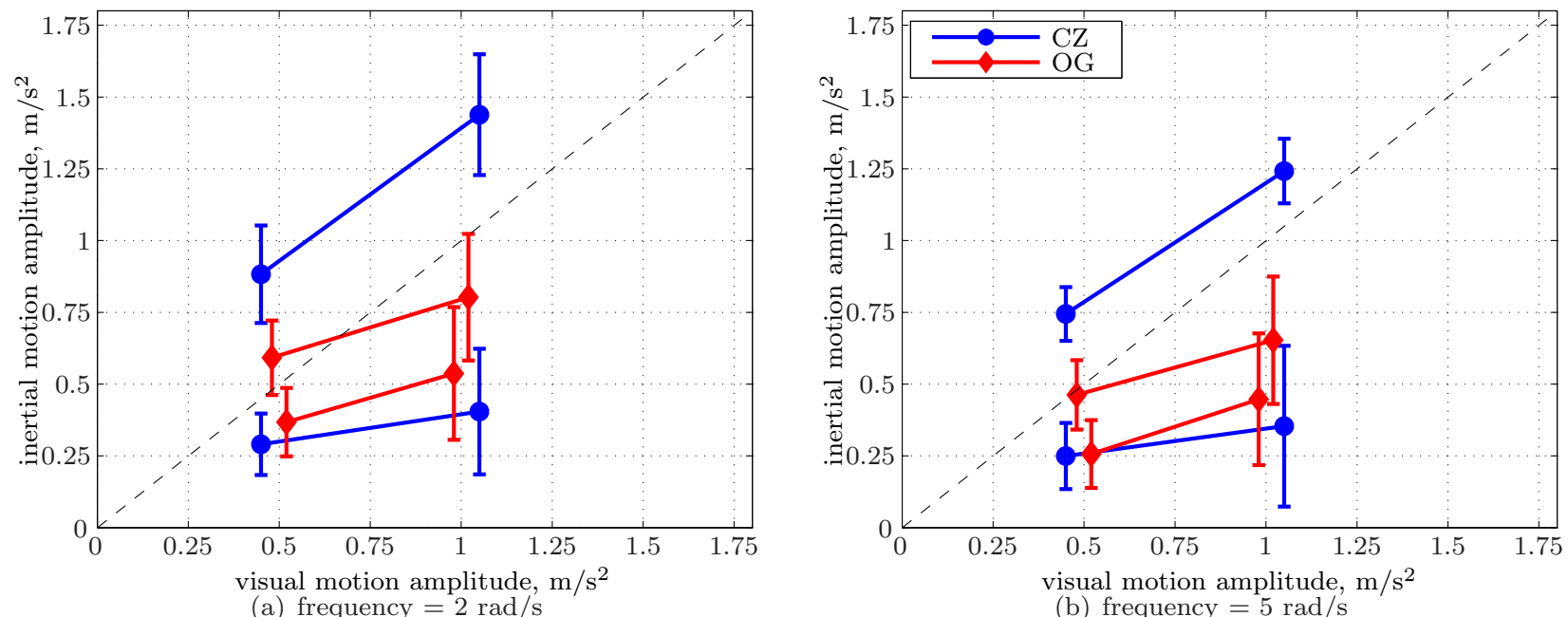

Figure 7. Mean coherence zones and optimal gain for two stimuli frequencies. The dashed line shows where the visual motion is equal to the inertial motion. The error bars indicate the $95 \%$ confidence intervals.

Figure 8 shows the mean PMZ and the ZW obtained from both the coherence zone and optimal gain. A repeated measures ANOVA was conducted to test whether the PMZ and ZW were different between the coherence zone and optimal gain. The results of the statistical analysis are shown in Table 3.

The PMZ was significantly influenced by the three tested independent variables (instructions, visual amplitude and stimuli frequency). From Figure 8(a) we observe that the PMZ values obtained during the coherence zone instructions are significantly higher than the PMZ values obtained during the optimal gain. Overall, the PMZ was higher for the experimental conditions with higher visual amplitude and lower for the conditions with higher frequency content. A significant interaction was found between the instructions and the visual amplitude. Figure 8(a) shows that the PMZ difference between the two instructions is smaller for the $0.5 \mathrm{~m} / \mathrm{s}^{2}$.

The ZW was significantly higher for the inertial accelerations obtained during the coherence zone instructions. This can be observed in Figure 8(b). Overall, the ZW was higher for the experimental conditions with higher visual amplitude and lower for the conditions with higher frequency content. The ZW was affected by an interaction between the measurement method and the visual amplitude. Figure 8(b) shows 


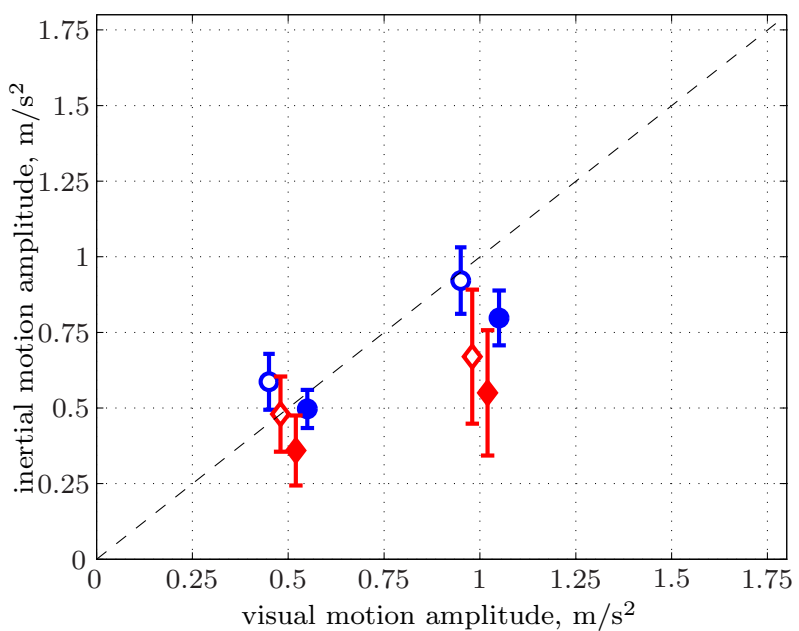

(a) Point of mean zone

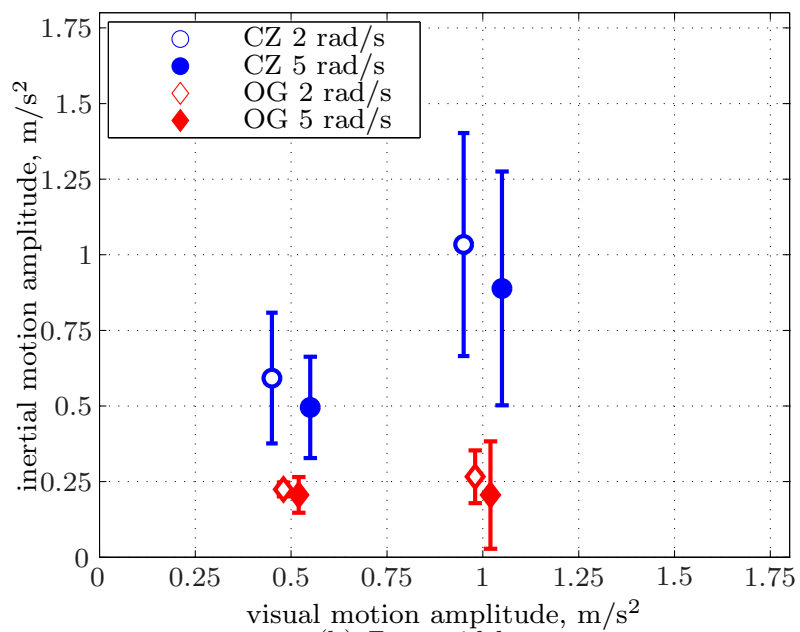

(b) Zone width

Figure 8. Mean PMZ and ZW for the coherence zone and optimal gain measurements. The dashed line in Figure 8(a) shows where the visual motion is equal to the inertial motion. The error bars indicate the $95 \%$ confidence intervals.

that the ZW increases with the visual amplitude for the measurements obtained during the coherence zone instructions whereas the ZW obtained with the optimal gain remains the same between the two different visual amplitudes.

Table 3. Repeated measures ANOVA results for the point of mean zone and the zone width.

\begin{tabular}{cccc}
\hline \hline Dependent Variables & Independent Variables & F-ratio & $p$ \\
\hline & Instruction & $\mathrm{F}(1,7)=12.07$ & 0.010 \\
PMZ & Amplitude & $\mathrm{F}(1,7)=54.76$ & 0.000 \\
& Frequency & $\mathrm{F}(1,7)=13.54$ & 0.008 \\
& Instruction * Amplitude & $\mathrm{F}(1,7)=7.59$ & 0.028 \\
\hline \multirow{4}{*}{ ZW } & Instruction & $\mathrm{F}(1,7)=16.78$ & 0.005 \\
& Amplitude & $\mathrm{F}(1,7)=16.08$ & 0.005 \\
& Frequency & $\mathrm{F}(1,7)=7.97$ & 0.026 \\
& Instruction * Amplitude & $\mathrm{F}(1,7)=12.81$ & 0.009 \\
\hline \hline
\end{tabular}

\section{III.B. The VMS simulator}

\section{III.B.1. Optimal Gain versus Coherence Zone}

Figure 9 shows the mean inertial accelerations obtained with the optimal gain and coherence zone methods in the VMS simulator. The trends found here are similar to the ones found in the CMF simulator (Figure 7). Again, the lower threshold seems comparable to the lower optimal gain. The upper threshold are higher than the upper optimal gain inertial accelerations. The PMZ and the ZW were used to compare the different instructions.

Figure 10 shows the PMZ and the ZW for the optimal gain and coherence zone obtained in the VMS simulator. A repeated measures ANOVA (Table 4) was conducted to test the effect of the independent variables in the PMZ and ZW.

The effects of the instructions, amplitude and frequency in the PMZ for the VMS simulator were the same as in the CMF simulator. Again, the PMZ was higher during the coherence zone measurements. Overall, the higher visual amplitudes increased the PMZ values whereas the higher frequency stimulus caused a decrease in the PMZ. Again, there was a significant interaction between the measurement method and the visual amplitude. Similar to the CMF results.

The ZW was significantly higher for the inertial acceleration values obtained with the coherence zone 


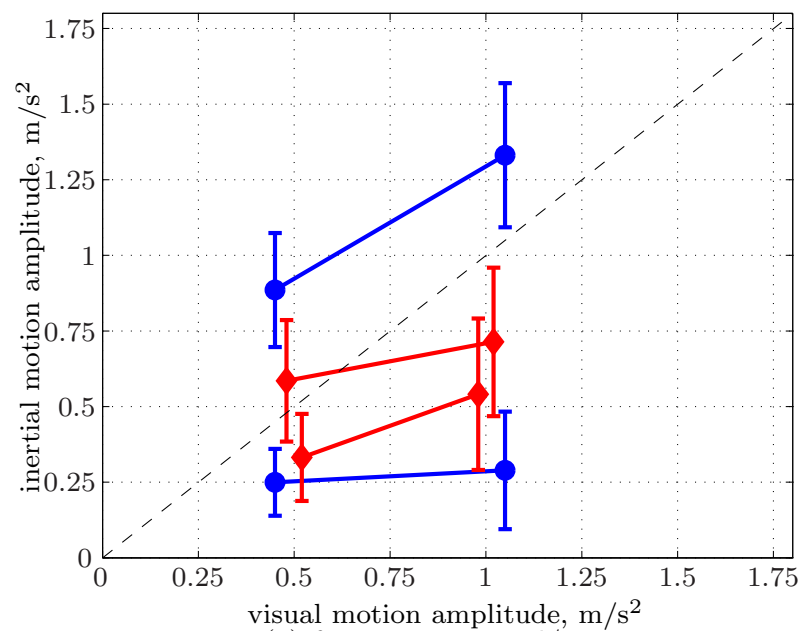

(a) frequency $=3 \mathrm{rad} / \mathrm{s}$

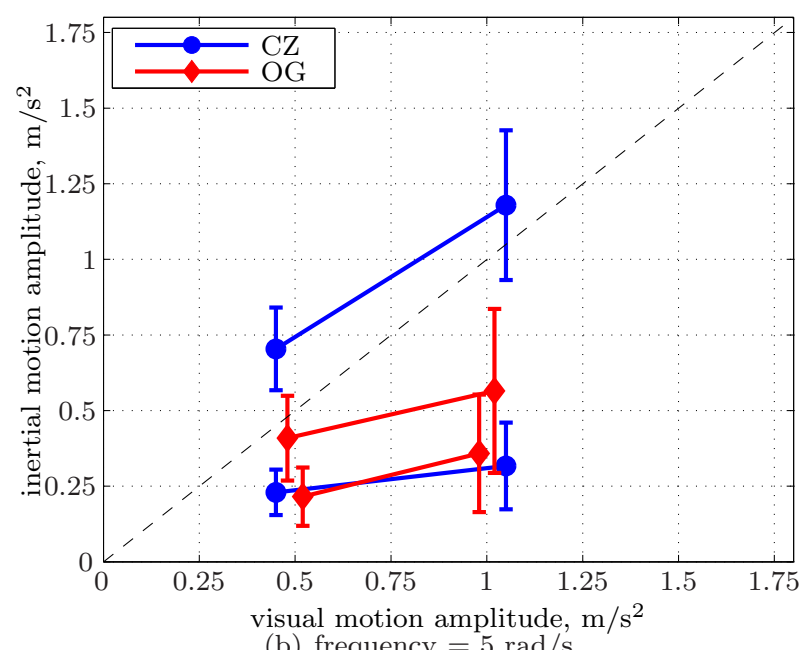

(b) frequency $=5 \mathrm{rad} / \mathrm{s}$

Figure 9. Mean Coherence zones and optimal gain for two stimuli frequencies. The dashed line shows where the visual motion is equal to the inertial motion. The error bars indicate the $95 \%$ confidence intervals.

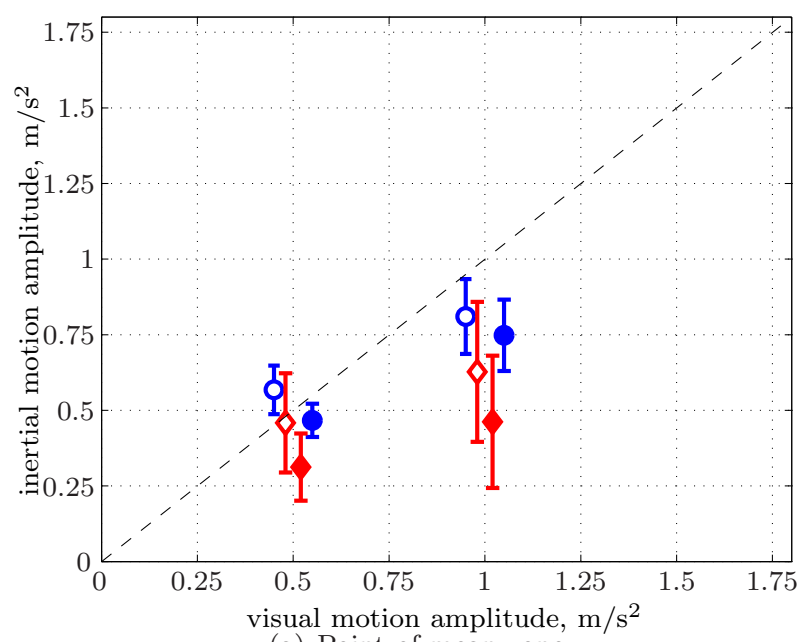

(a) Point of mean zone

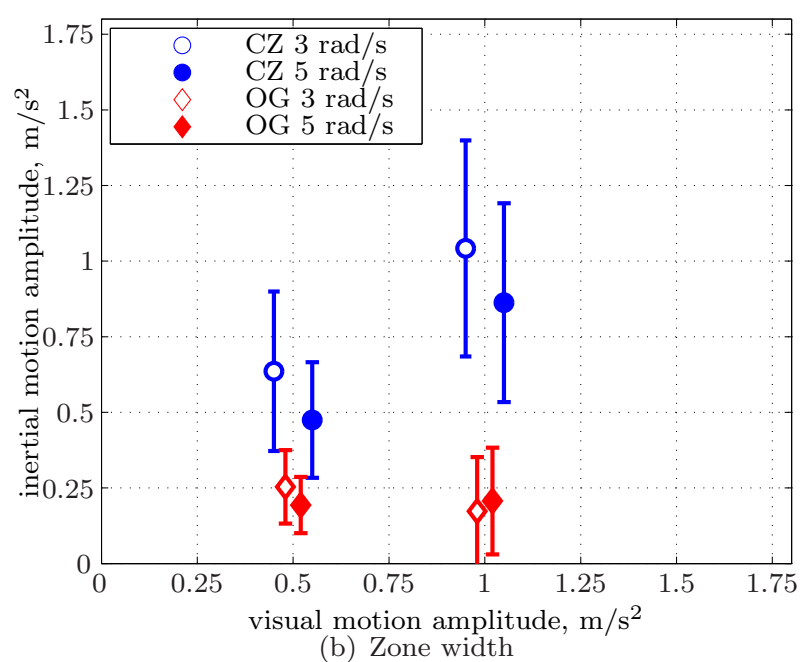

(b) Zone width

Figure 10. Mean PMZ and $\mathrm{ZW}$ for the coherence zone and optimal gain obtained in the VMS simulator. The dashed line in Figure 10(a) shows where the visual motion is equal to the inertial motion. The error bars indicate the $95 \%$ confidence intervals. 
measurements. Overall, the inertial acceleration values were higher for the conditions with an higher visual amplitude and lower for the conditions with higher frequency content. As was seen in the CMF, there was also a significant interaction between the instruction and the visual amplitude in the VMS. Additionally, in the VMS we found a significant interaction between the instruction and the frequency of the stimulus. If we pool the amplitudes of Figure 10(b), the ZW of the coherence zone is higher for the $3 \mathrm{rad} / \mathrm{s}(0.84$ $\left.\mathrm{m} / \mathrm{s}^{2}\right)$ than for the $5 \mathrm{rad} / \mathrm{s}(0.67)$. For the ZW of the optimal gain the value of the $2 \mathrm{rad} / \mathrm{s}\left(0.21 \mathrm{~m} / \mathrm{s}^{2}\right)$ is approximately the same of the $5 \mathrm{rad} / \mathrm{s}\left(0.20 \mathrm{~m} / \mathrm{s}^{2}\right)$.

Table 4. Repeated measures ANOVA results for the point of mean zone and the zone width in the VMS simulator.

\begin{tabular}{cccc}
\hline \hline Dependent Variables & Independent Variables & F-ratio & $p$ \\
\hline \multirow{2}{*}{ PMZ } & Instruction & $\mathrm{F}(1,7)=8.59$ & 0.022 \\
& Amplitude & $\mathrm{F}(1,7)=32.61$ & 0.001 \\
& Frequency & $\mathrm{F}(1,7)=16.68$ & 0.005 \\
& Instruction * Amplitude & $\mathrm{F}(1,7)=6.85$ & 0.035 \\
\hline \multirow{2}{*}{$\mathrm{ZW}$} & Instruction & $\mathrm{F}(1,7)=12.98$ & 0.009 \\
& Amplitude & $\mathrm{F}(1,7)=19.70$ & 0.003 \\
& Frequency & $\mathrm{F}(1,7)=8.98$ & 0.020 \\
& Instruction * Amplitude & $\mathrm{F}(1,7)=33.32$ & 0.001 \\
& Instruction * Frequency & $\mathrm{F}(1,7)=7.90$ & 0.026 \\
\hline \hline
\end{tabular}

\section{III.C. CMF versus VMS}

Figure 11 shows the mean PMZ and ZW in both simulators. Only the results of the $5 \mathrm{rad} / \mathrm{s}$ experimental conditions were used since this was the only condition that was performed in both simulators. A statistical analysis was performed to test whether the simulator had an effect on the PMC or the CZW. The repeated measures ANOVA results are shown in Table 5.

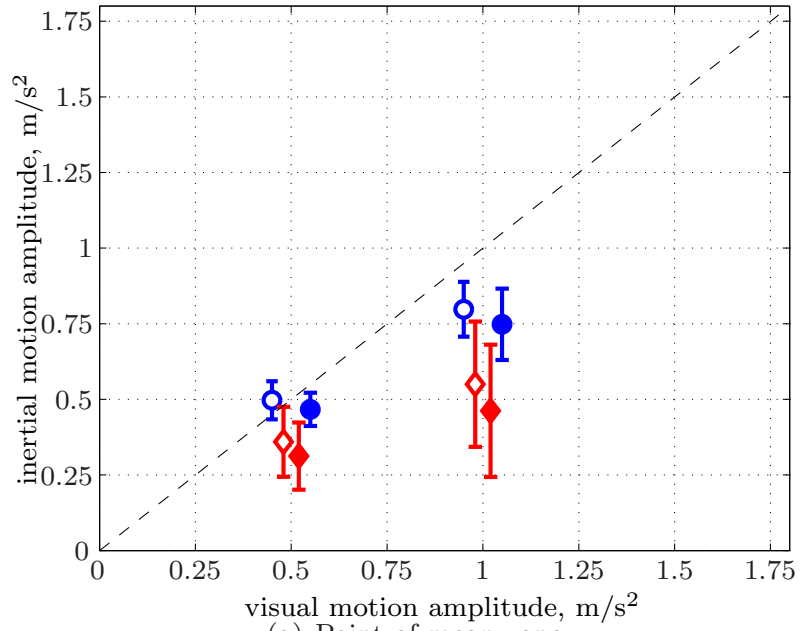

(a) Point of mean zone

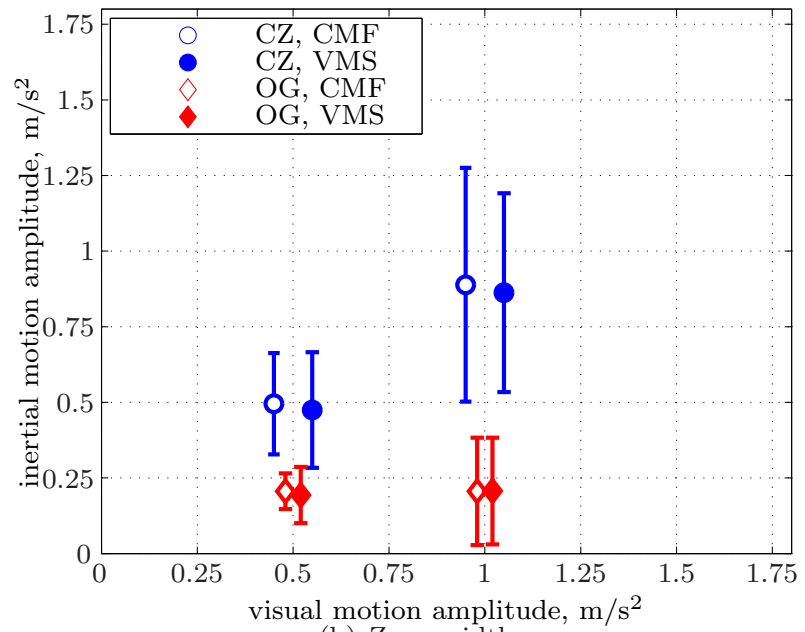

(b) Zone width

Figure 11. Comparison of the Mean PMZ and ZW between the CMF and VMS simulators. The dashed line in Figure 11(a) shows where the visual motion is equal to the inertial motion. The error bars indicate the $95 \%$ confidence intervals.

The statistical analysis showed no difference in the PMZ and the ZW results found in the different simulators. From Figure 11 it is observed that the PMC and the CZW were approximately the same between simulators. The significant effects shown in Table 5 of the other independent variables are similar to what was reported in the previous sections and therefore will not be described further. 
Table 5. Repeated measures ANOVA results for the point of mean zone and the zone width.

\begin{tabular}{cccc}
\hline \hline Dependent Variables & Independent Variables & F-ratio & $p$ \\
\hline & Simulator & $\mathrm{F}(1,7)=0.63$ & 0.453 \\
PMZ & Instruction & $\mathrm{F}(1,7)=29.67$ & 0.001 \\
& Amplitude & $\mathrm{F}(1,7)=79.42$ & 0.000 \\
& Instruction * Amplitude & $\mathrm{F}(1,7)=7.37$ & 0.030 \\
\hline \multirow{3}{*}{$\mathrm{ZW}$} & Simulator & $\mathrm{F}(1,7)=0.04$ & 0.857 \\
& Instruction & $\mathrm{F}(1,7)=16.93$ & 0.004 \\
& Amplitude & $\mathrm{F}(1,7)=22.94$ & 0.002 \\
& Instruction * Amplitude & $\mathrm{F}(1,7)=14.00$ & 0.007 \\
\hline \hline
\end{tabular}

\section{Discussion}

\section{IV.A. Coherence Zone versus Optimal Gain}

In this experiment we measured perception coherence zones and perceived optimal gain. As seen in a previous study, ${ }^{10}$ the optimal gain measurements did not result in one single value, but in a zone bounded by an upper and a lower limit. For comparison purposes, this optimal gain zone was defined by the point of mean optimal gain (PMO) and the optimal gain width (OGW) and the coherence zone, similar to what was done in other studies, ${ }^{11,12}$ was defined by the point of mean coherence (PMC) and the coherence zone width $(\mathrm{CZW})$.

The perception coherence zones were different from the optimal gain zones. In general, the optimal gain zone was contained within the coherence zone, as if there was a subset of coherent inertial and visual cues that were perceived as a better match. Together, the coherence and optimal gain zones define three regions: within the optimal gain zone, outside the optimal gain zone and within the coherence zone, and outside the coherence zone. These regions can be considered as a gradient of accepted motion ranging from best (within the optimal gain) to worst (outside the coherence zone) motion.

Nevertheless, the question remains to what perception mechanism allows subjects to distinguish between a coherent and an optimal region. Two different assumptions were made to attempt to explain the differences.

The different instructions could have created different paradigms when measuring the two zones. For the coherence zone subjects start with similar inertial and visual amplitudes and their objective is to increase or decrease the inertial amplitude until a difference between the inertial and visual amplitude is noticed. Conversely, for the for the optimal gain subjects start from different inertial and visual amplitudes and their objective is to change the inertial amplitude until the two stimulus are perceived as equal. Although both tasks sound similar, asking for differences between two stimulus seems to create a different result than asking if two stimulus are equal.

What could also have led to the differences between the two zones was what may be called as "tuning for comfort". Higher inertial amplitudes can be more uncomfortable for the subjects than lower inertial amplitudes. For the optimal gain subjects are asked to find the best match between visual and inertial information. It could happen that the best match is not where the visual amplitude is equal to the motion amplitude but the most comfortable, less arousing motion amplitude that is still perceived as coherent with the visual motion. Such approach would lead to a PMO lower than the PMC since for the coherence zone subjects are looking for the boundary of the coherence and not for the more comfortable motion.

This explanation agrees with the found PMOs which were significantly lower than the PMCs. The PMC was generally closer to the one-to-one line than the PMO. Careful observation of the limits for both zones shows that this difference was caused mainly by the upper threshold and upper optimal gain measurement since the lower bounds of both zones were similar. The coherence zone upper threshold is always above the one-to-one line whereas the upper optimal gain crosses the one-to-one line for the $2 \mathrm{rad} / \mathrm{s}$ conditions and is bellow the one-to-one line for the $5 \mathrm{rad} / \mathrm{s}$ conditions. It appears that the optimal gain zone excludes only the higher inertial amplitudes within the coherence zone.

The PMO and PMC increase with the visual stimulus amplitude and decreased with the stimulus frequency. These trends have been found before for optimal gain measurements in sway ${ }^{10}$ and coherence zone measurements in yaw. ${ }^{11,12}$ The effect of stimulus frequency on the coherence zone was tentatively explained 
with the influence of the semi-circular canals dynamics, ${ }^{11,12}$ which have a higher gain in velocity at higher frequencies. This higher gain may not be accounted for during the internal comparison of inertial and visual cues, leading subjects to down tune the inertial cues at higher frequencies. In the present study, since the tested motion was sway, a similar explanation might be found in the sensitivity to jerk. For the same acceleration amplitude, when the frequency increases the jerk also increases. Grant and Haycock ${ }^{13}$ showed that the concept of motion strength is a relation between the jerk and acceleration of a inertial motion signal. It could have happened that subjects lowered the upper threshold of the conditions with higher frequency so as to lower the jerk and therefore lower the perceived motion strength.

The PMO and PMC increase with visual stimulus amplitude but not as much as it would be expected. For the lowest amplitude the PMCs and PMOs are close to the one-to-one line and for the highest amplitude they are both below this line. This decrease with respect to the one-to-one line is more evident for the PMO. If these results were to be used in motion filter tuning, the motion gain, i.e. the ratio between the inertial and visual motion, should generally be smaller than one, especially for the highest amplitude. This supports the findings of other studies ${ }^{6-10}$ where a motion gain of one was judged as too strong. A reason for one-to-one motion to be judged too strong in a simulation environment may be the quality of the visual display. ${ }^{6,7}$ Such hypotheses still needs to be validated. However, a recent study by Zaal et al. ${ }^{14}$ showed that even when pilots had similar visual conditions in the simulator and in the aircraft, pilots showed a different control behavior in a roll tracking task between the aircraft and the simulator with a one-to-one motion condition. Other studies point out that these differences may be due to environmental factors, such as vibrations, or pilot centered factors, such as expectations. ${ }^{15}$

The OGW was significantly lower than the CZW. The CZW was affected by the visual amplitude and frequency and, as found previously for yaw motion, ${ }^{11,12}$ was coupled to the PMCs. A higher PMC corresponds to a higher CZW value and vice-versa. Unlike the CZW, the OGW was unaffected by these two independent variables. The OGW remained approximately the same (around $0.23 \mathrm{~m} / \mathrm{s}^{2}$ ) for all the experimental conditions. This is perhaps the most remarkable difference found between the optimal gain zone and the coherence zone. Since the lower thresholds of the coherence and optimal gain zones varied comparatively less with frequency than the upper thresholds, the main difference in the zones width may be related to the upper limits. As seen before, the upper limits of the optimal gain zone were significantly lower than the coherence zone ones. Again, this result may be related to a "tune for comfort" effect, although no reason could be found to why this would lead to a OGW unaffected by visual stimulus amplitude or frequency.

Further research should be done to investigate if the constant OGW results found for the tested amplitudes also occurs at higher amplitudes, and if comfort has an effect on the optimal gain measurements. For applications to motion simulation, it is also important to know what are the consequences for perception and behavior of moving out of the optimal gain zone while remaining within the coherence zone.

\section{IV.B. CMF versus VMS}

Both the coherence zone and the optimal gain showed no significant difference between the VMS and the CMF simulator. The simulators had comparable visual systems but different motion platforms. The VMS had a lower signal-to-noise $(\mathrm{S} / \mathrm{N})$ ratio. However, the inertial gains obtained for the optimal gain and coherence zone measurements were the same for both simulators. The PMZ results, especially the PMO, was slightly lower for the VMS simulator. However, this tendency was not statistically significant. Nevertheless, final conclusions should not be made based only on the $5 \mathrm{rad} / \mathrm{s}$ conditions that were tested in both simulators. In the VMS, there is a significant interaction between the instructions and the frequency that it is not found in the CMF. Therefore, there is indication that the measured optimal gain and coherence zones may have been differently affected by frequency in the two simulators. However this cannot be tested in a ANOVA because in the CMF the lower frequency was $2 \mathrm{rad} / \mathrm{s}$ whereas in the VMS it was $3 \mathrm{rad} / \mathrm{s}$.

Two different results could be expected when comparing simulators with different motion quality. We could expect a down-tuning of the inertial motion in the VMS, since the motion platform is noisier and more motion, whether or not in the desired degree-of-freedom, is presented to the subjects. On the other hand, especially for the lower thresholds, one could expect subjects to require higher inertial motion amplitudes in the VMS to facilitate the perception of the sway motion above motion the motion in other degrees-offreedom, caused by the cross-coupling. The slight lower PMZ for the VMS simulator indicates a tendency for the down-tuning hypothesis. However, one should be careful when drawing any conclusions from the data since this tendency was not statistically significant. 


\section{Conclusion}

This study showed that the coherence zone is different from the optimal gain zone. The optimal gain zone is within the coherence zone. Both the coherence and the optimal gain zone were affected by the visual amplitude and frequency. The PMC and CZW where significantly higher than the PMO and the OGW. Unlike the other measurements, the OGW was not affected by the visual amplitude or frequency. The PMC was closer to the one-to-one line than the PMO. This could be an indication that the optimal gain instructions are related with "tuning for comfort".

There were no significant differences between both simulators for the measured coherence and optimal gain zones. However only the $5 \mathrm{rad} / \mathrm{s}$ condition was tested in both simulators. Our results suggest that differences between the two simulators may be found at lower visual frequencies.

\section{Acknowledgments}

The first author is supported by TNO Defence, Security and Safety. The second author is supported by a Toptalent grant from the Netherlands Organisation for Scientific Research (NWO).

\section{References}

\footnotetext{
${ }^{1}$ Nahon, M. A. and Reid, L. D., "Simulator Motion-Drive Algorithms: A Designer's Perspective," Journal of Guidance, Control and Dynamics, Vol. 13, No. 2, 1990, pp. 356-362.

${ }^{2}$ Hosman, R. J. A. W., Pilot's perception and control of aircraft motions, Ph.D. thesis, Delft University of Technology, 1996.

${ }^{3}$ van der Steen, H., Self-Motion Perception, Ph.D. thesis, Delft University of Technology, 1998.

${ }^{4}$ Groen, E. L. and Bos, J. E., "Simulator Sickness Depends on Frequency of the Simulator Motion Mismatch: An Observation," PRESENCE: Teleoperators and Virtual Environments, Vol. 17, No. 6, 2008, pp. 584-593.

${ }^{5}$ Groen, E. L. and Bos, J. E., "Identification of "Bad Simulator Motion"," DSC 2008 Europe, Monaco, January-February 2008, pp. 237-245.

${ }^{6}$ Groen, E. L., Clari, M. S. V. V., and Hosman, R. J. A. W., "Evaluation of perceived motion during a simulated takeoff run," Journal of Aircraft, Vol. 38, No. 4, 2001, pp. $600-606$.

${ }^{7}$ Groen, E. L., Clari, M. S. V. V., and Hosman, R. J. A. W., "Perception model analysis of flight simulator motion for a decrab maneuver," Journal of Aircraft, Vol. 44, No. 2, 2007, pp. 427 - 435.

${ }^{8}$ Feenstra, P., Wentink, M., Correia Grácio, B. J., and Bles, W., "Effect of Simulator Motion Space on Realism in the Desdemona Simulator," DSC 2009 Europe, Monaco, 2009.

${ }^{9}$ Pretto, P., Nusseck, H. G., Teufel, H. J., and Bülthoff, H. H., "Effect of lateral motion on driver's performance in the MPI motion simulator," DSC 2009 Europe, Monaco, 2009.

${ }^{10}$ Correia Grácio, B. J., van Paassen, M. M., Mulder, M., and Wentink, M., "Tuning of the lateral specific force gain based on human motion perception in the Desdemona simulator," AIAA Modeling and Simulation Technologies Conference and Exhibit, No. AIAA 2010-8094, Toronto, Ontario Canada, August 2010.

${ }^{11}$ Valente Pais, A. R., van Paassen, M. M., Mulder, M., and Wentink, M., "Perception of Combined Visual and Inertial Low-Frequency Yaw Motion," AIAA Modeling and Simulation Technologies Conference and Exhibit, No. AIAA 2010-8093, Toronto, Ontario Canada, August 2010.

${ }^{12}$ Valente Pais, A. R., van Paassen, M. M., Mulder, M., and Wentink, M., "Perception Coherence Zones in Flight Simulation," Journal of Aircraft, Vol. 47, No. 6, 2010, pp. 2039-2048.

${ }^{13}$ Grant, P. R. and Haycock, B., "Effect of Jerk and Acceleration on the Perception of Motion Strength," Journal of Aircraft, Vol. 45, No. 4, 2008, pp. $1190-1197$.

${ }^{14}$ Zaal, P. M. T., Pool, D. M., van Paassen, M. M., and Mulder, M., "Comparing Multimodal Pilot Pitch Control Behavior Between a Simulator and Real Flight," AIAA Modeling and Simulation Technologies Conference and Exhibit, Portland, OR, August 2011 (submitted for publication).

${ }^{15}$ Schroeder, J. A. and Grant, P. R., "Pilot Behavioral Observations in Motion Flight Simulation," AIAA Modeling and Simulation Technologies Conference and Exhibit, No. AIAA 2010-8353, Toronto, Ontario Canada, August 2010.
} 